Recherches en didactique des langues et des cultures

Les cahiers de l'Acedle

13-3 | 2016

Travailler sur et à partir de textes écrits en classe de langue étrangère

\title{
La correction de l'expression écrite en langue étrangère et son exploitation pédagogique
}

Expliciter l'implicite?

Kevin Noiroux et Germain Simons

\section{OpenEdition}

\section{Journals}

Édition électronique

URL : http://journals.openedition.org/rdlc/1302

DOI : $10.4000 /$ rdlc. 1302

ISSN : 1958-5772

Éditeur

ACEDLE

\section{Référence électronique}

Kevin Noiroux et Germain Simons, «La correction de l'expression écrite en langue étrangère et son exploitation pédagogique », Recherches en didactique des langues et des cultures [En ligne], 13-3 | 2016, mis en ligne le 31 décembre 2016, consulté le 30 avril 2019. URL : http://journals.openedition.org/ rdlc/1302 ; DOI : 10.4000/rdlc.1302

Ce document a été généré automatiquement le 30 avril 2019.

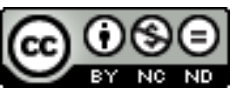

Recherches en didactique des langues et des cultures is licensed under a Creative Commons AttributionNonCommercial-NoDerivatives 4.0 International License 


\title{
La correction de l'expression écrite en langue étrangère et son exploitation pédagogique
}

\author{
Expliciter l'implicite?
}

\author{
Kevin Noiroux et Germain Simons
}

\section{Introduction}

1 L'enseignant est confronté à toute une série de choix pédagogiques et didactiques qui influencent peu ou prou la performance de l'apprentissage de ses élèves. Notre recherche se penche sur les choix qui ont trait à la régulation des apprentissages. Il s'agit d'un champ de recherche complexe, car il comporte de nombreux gestes didactiques qui s'opèrent dans différents modes et modalités (par exemple l'écrit et l'oral, seul ou collégialement). Plus spécifiquement, nous nous intéressons ici à la correction des erreurs produites dans le cadre de la rédaction d'un texte en langue étrangère. Pour corriger les erreurs de ses élèves, l'enseignant dispose de plusieurs choix : localiser l'erreur ou laisser l'apprenant la trouver, expliquer l'erreur, la corriger...

2 Les recherches scientifiques récentes qui portent sur la correction des erreurs tendent à conclure que cette dernière option, appelée correction directe - c'est-à-dire fournir à l'apprenant une version correcte de son énoncé erroné - est plus efficace que les autres formes de correction. Cependant, bien que les chercheurs émettent des hypothèses pertinentes sur l'efficacité accrue de cette forme de correction sur les autres, leur angle d'approche reste comparatiste : ils n'analysent pas les tenants et aboutissants afférents à l'utilisation de la correction directe. Aucune méthodologie n'est mise en place pour mesurer le taux de compréhension des erreurs corrigées.

3 Pour accomplir cette tâche, nous avons réalisé une recherche exploratoire qui vise à déterminer le taux de compréhension des erreurs et de leur correction comme mesure de l'efficacité de ce mode de correction : si les élèves déclarent et prouvent qu'ils ont compris l'erreur corrigée, la correction peut être jugée efficace. Dans le cas contraire, 
l'enseignant n'a pas atteint son but. Plus le nombre d'erreurs non comprises est élevé, plus la correction directe est un mode de régulation limité.

Tous ces choix s'inscrivent dans un contexte particulier qui influe sur les options dont l'enseignant dispose, et ce pour des raisons tant institutionnelles que pragmatiques. Le contexte de notre recherche est celui de la Fédération Wallonie-Bruxelles (l'ancienne Communauté française de Belgique) qui, pour diverses raisons, limite les options à la disposition des enseignants.

Cette contribution s'articule autour de quatre grandes parties.

Dans la partie 1 destinée au cadrage théorique, nous situons d'abord l'acte d'évaluation de l'expression écrite dans un processus pédagogique plus large et précisons la focale que nous avons choisie dans cette contribution, en l'occurrence la correction du texte par l'enseignant et le traitement qui peut être fait de cette copie annotée en classe. Ensuite, nous passons en revue la recherche sur les feedbacks correctifs en expression écrite en proposant une définition et une typologie de ces feedbacks et en argumentant l'intérêt de cet outil pour améliorer la maitrise de la langue étrangère.

Dans la partie 2, nous évoquons le contexte spécifique de l'enseignement des langues en Fédération Wallonie-Bruxelles (désormais FWB) dans lequel nous avons effectué notre recherche exploratoire, car ce contexte a un impact sur les choix posés par l'enseignant.

Dans la partie 3, nous présentons une recherche exploratoire réalisée dans une classe de $5^{e}$ année d'anglais première langue étrangère (entre 16 et 17 ans) de l'enseignement secondaire général. Cette recherche a pour objectif de mieux appréhender ce que les élèves comprennent de leurs erreurs sur la base des corrections directes fournies par l'enseignant sur les copies, et du dispositif mis en place suite à la distribution de celles-ci. Les résultats nous permettent de conclure à une efficacité relative et limitée de la correction directe.

9 Dans la partie 4, nous procédons à une synthèse de l'article et proposons quelques pistes de solution pour tenter de résoudre les problèmes soulevés préalablement.

\section{Cadrage théorique : régulation des apprentissages et feedback correctif}

10 Allal définit la régulation des apprentissages «[...] en termes d'une succession d'opérations visant à fixer un but et orienter l'action vers celui-ci ; contrôler la progression de l'action vers le but; assurer un retour sur l'action (un feedback, une rétroaction) ; confirmer ou réorienter la trajectoire de l'action, et/ou redéfinir le but » (2007 : 8). Parmi ses nombreuses missions, l'enseignant doit enseigner à ses élèves comment rédiger un texte dans une langue formellement correcte. Lorsque les élèves commettent des erreurs, il doit par conséquent réguler leur apprentissage pour qu'ils ne les commettent plus à l'avenir. Dans le cadre de la production de textes, il est très fréquent que l'enseignant annote la copie de l'élève au moyen de feedbacks correctifs écrits (désormais FCE).

11 L'annotation de la copie de l'élève par l'enseignant peut prendre des formes très différentes, de même que le traitement qui en est fait ultérieurement par le professeur et les élèves. Comme le synthétise la figure 1 , on peut identifier quatre grandes phases dans le processus de production et de traitement de la copie écrite initiale de l'élève. 
Figure 1 - Grandes étapes de la démarche de traitement de la copie d'expression écrite initiale par l'enseignant et les élèves $(*)$

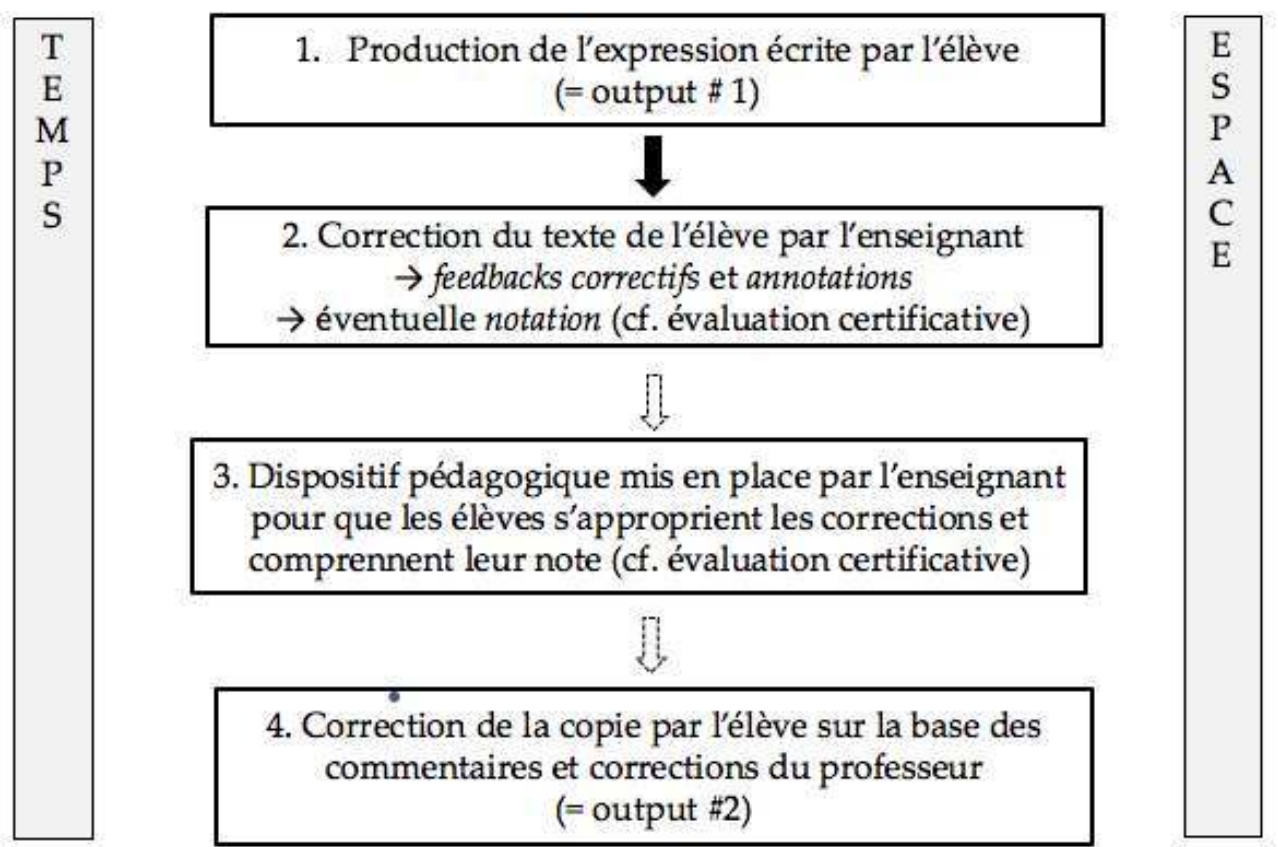

(*) Les flèches hachurées indiquent que les étapes qui suivent ne sont pas nécessairement mises en pratique dans toutes les classes.

Dans le cadre de cet article, nous nous focalisons principalement sur les étapes 2 et 3.

\section{Le feedback correctif écrit}

13 Le FCE indique à l'élève qu'il a commis une erreur de langue (Russell \& Spada, 2006 : 134). Son but est d'attirer l'attention de l'élève sur son utilisation erronée de la langue cible pour lui permettre de restructurer son interlangue ${ }^{1}$ et, in fine, de ne plus commettre la même erreur. Le FCE cible les erreurs lexicogrammaticales (morphologiques, syntaxiques, lexicales, d'orthographe, etc.) ; les erreurs plus macro (p. ex. de structuration, de cohérence et de pertinence du contenu) relèvent du feedback sur l'écrit (feedback on writing) et ne sont pas traitées dans cet article, car elles font appel à des processus cognitifs différents.

14 Les dernières recherches sur le FCE, que nous synthétisons ci-dessous (cf. 1.3.), tendent à conclure que la correction directe, qui consiste à fournir la forme correcte de l'erreur, est plus efficace que la correction indirecte, où il est seulement fait mention à l'élève qu'il a commis une erreur. Mais qu'en est-il de la compréhension effective du FCE direct ? En effet, la correction directe a beau s'avérer plus performante que son homologue indirect, cela n'implique pas qu'elle soit nécessairement performante, c'est-à-dire qu'elle permette à tous les élèves de comprendre la majorité de leurs erreurs. Si un élève comprend $10 \%$ de ses erreurs quand elles sont soulignées, mais $20 \%$ quand elles sont soulignées et corrigées, le deuxième mode de correction est certes plus performant que le premier, mais il n'en est pas performant pour autant puisque l'élève ne comprend qu'une erreur sur cinq. L'objet de notre recherche est précisément de mesurer ce taux de compréhension (voir point 3). 

étrangère indiquent que le FCE peut contribuer à restructurer l'interlangue de l'élève. Parmi ceux-ci, nous en épinglons deux qui nous paraissent fondamentaux. Le premier est que l'exposition à des énoncés erronés (negative evidence) qui informent l'élève qu'il dévie de la norme, peut être bénéfique à l'acquisition d'une langue étrangère (Gass, 1988 ; White, 1991). Le second, celui d'"attention" développé par Schmidt (1990, 1995, 2001), postule que pour acquérir une langue étrangère, il faut prêter attention aux éléments qui la constituent pour qu'ils s'intègrent à l'interlangue. Concrètement, l'apprenant, grâce au FCE, prête une attention sélective à son énoncé corrigé, ce qui lui permet de percevoir ( notice) le fossé (gap) entre les versions erronée et correcte de l'énoncé et, ce faisant, de prendre conscience (awareness) de ses erreurs et de faire évoluer son interlangue.

'utilisation du FCE semble être une pratique fondée, courante et perçue par les enseignants et les élèves comme indispensable à l'acquisition de la langue étrangère, elle a néanmoins été questionnée par quelques chercheurs, le plus influent d'entre eux étant Truscott. Dans son article "The Case Against Grammar Correction in L2 Writing Classes" (1996), il affirme qu'il faut renoncer à la correction grammaticale pour trois raisons : (1) de nombreuses recherches montrent que le FCE est inefficace ; (2) tant pour des raisons théoriques que pratiques, on s'attend à ce que le FCE soit inefficace ; et (3) le FCE produit des effets contreproductifs sur l'apprentissage de la langue étrangère. Ces postulats ont poussé de nombreux chercheurs à investiguer l'efficacité du FCE (Bitchener, 2008 ; Bitchener, Young \& Cameron, 2005 ; Chandler, 2003 ; Ellis, Loewen \& Erlam, 2006 ; Ferris, 1999, 2004, 2006 ; Ferris \& Roberts, 2001 ; Sheen, 2010, 2011 ; Sheen \& Ellis, 2011). De ces recherches (cf. 1.3.), on conclut que le FCE peut améliorer la correction lexicogrammaticale de l'écrit suivant.

\section{Typologie des FCE}

Notre problématique se centre sur les FCE comme outil de régulation. L'enseignant dispose de différentes stratégies pour indiquer à l'élève qu'il a commis une erreur. La synthèse suivante s'inspire des travaux de Sheen \& Ellis (2011 : 594).

Tableau 1 - Typologie des feedbacks correctifs écrits.

\begin{tabular}{|c|c|c|c|c|c|c|}
\hline \multirow[t]{2}{*}{ Types } & \multirow[t]{2}{*}{ Exemples } & \multirow[t]{2}{*}{ Explications } & \multicolumn{4}{|c|}{ «Caractéristiques» } \\
\hline & & & $\begin{array}{l}\text { direct ou } \\
\text { indirect }\end{array}$ & $\begin{array}{l}\text { localisé } \\
\text { ou non } \\
\text { localisé }\end{array}$ & $\begin{array}{l}\text { implicite ou } \\
\text { explicite }\end{array}$ & $\begin{array}{l}\text { global } \\
\text { ou local }\end{array}$ \\
\hline 1. & $\begin{array}{l}\mathrm{X} \mid \mathrm{I} \text { write to } \\
\text { you because... }\end{array}$ & $\begin{array}{l}\text { On indique dans la marge } \\
\text { (en l'occurrence au moyen } \\
\text { de croix, de barres ou de } \\
\text { chiffres) le nombre } \\
\text { d'erreurs que la ligne } \\
\text { contient. }\end{array}$ & indirect & $\begin{array}{l}\text { non } \\
\text { localisé }\end{array}$ & implicite & $\begin{array}{l}\text { global } \\
\text { ou local }\end{array}$ \\
\hline
\end{tabular}




\begin{tabular}{|c|c|c|c|c|c|}
\hline 2. & 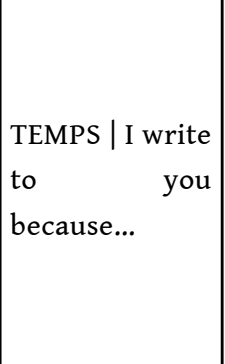 & $\begin{array}{l}\text { On indique dans la marge } \\
\text { le nombre et le type } \\
\text { d'erreurs que la ligne } \\
\text { contient, par exemple au } \\
\text { moyen du code "temps", } \\
\text { qui indique qu'une erreur } \\
\text { de temps a été commise } \\
\text { dans la ligne qui le suit }{ }^{2} \text {. }\end{array}$ & indirect & $\begin{array}{l}\text { non } \\
\text { localisé }\end{array}$ & \\
\hline 3. & $\begin{array}{l}\text { I write to you } \\
\text { because... }\end{array}$ & $\begin{array}{l}\text { On localise l'erreur, par } \\
\text { exemple en la soulignant. }\end{array}$ & indirect & localisé & \\
\hline 4. & $\begin{array}{l}\text { TEMPS } \\
\text { I write to you } \\
\text { because... }\end{array}$ & $\begin{array}{l}\text { On localise l'erreur et on } \\
\text { précise son type. }\end{array}$ & indirect & localisé & \\
\hline 5. & $\begin{array}{l}\text { I'm writing } \\
\text { I rrite to you } \\
\text { because... }\end{array}$ & $\begin{array}{l}\text { On localise et corrige } \\
\text { l'erreur. }\end{array}$ & direct & localisé & \\
\hline 6. & $\begin{array}{l}\text { I } \underline{\text { write }}(1) \text { to } \\
\text { you because... } \\
\text { (1) action } \\
\text { temporaire => } \\
\text { présent } \\
\text { continu }\end{array}$ & $\begin{array}{l}\text { On localise et explique } \\
\text { l'erreur. }\end{array}$ & indirect & localisé & \\
\hline 7. & 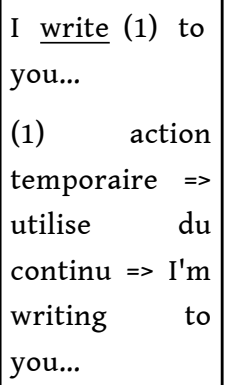 & $\begin{array}{l}\text { On localise, explique et } \\
\text { corrige l'erreur. }\end{array}$ & direct & localisé & explicite \\
\hline
\end{tabular}

18 Chaque type possède les traits suivants : le FCE est (1) direct si la correction est fournie, indirect si elle ne l'est pas ; (2) localisé si le mot erroné est précisément identifié, non localisé s'il ne l'est pas précisément; (3) explicite si la correction est pourvue d'explications métalinguistiques, implicite s'il n'y a aucune explication. Le dernier trait concerne la stratégie de correction de la copie entière de l'élève : (4) le FCE est global s'il cible toutes les erreurs de langue, local s'il cible un ou deux types d'erreurs (p. ex. uniquement les erreurs de temps).

Chaque type de FCE traduit un postulat didactique quant à son efficacité. Par exemple, ne pas corriger l'erreur ${ }^{3}$ repose sur le postulat selon lequel l'investissement de l'élève dans la découverte de son erreur lui permet de mieux assimiler la forme correcte que si cette dernière lui est fournie. 


\section{Le feedback correctif direct}

Déjà avant la publication très controversée de l'article de Truscott (1996), de nombreux chercheurs ont tenté d'identifier le type de FCE le plus efficace (cf. tableau 1). Entre le FCE direct (type 5) et les FCE indirects (types 1-4), les études les plus récentes indiquent que le FCE direct est le plus performant, du moins sur le long terme (Chandler, 2003 ; C. G. Van Beuningen, De Jong \& Kuiken, 2008 ; Catherine G Van Beuningen, De Jong \& Kuiken, 2012), à l'exception notable de la recherche de Ferris (2006) pour qui le FCE indirect (type 4) est plus performant que la correction directe (type 5). Il faut cependant relever que l'efficacité des FCE explicites (types 6-7) est le parent pauvre de la recherche sur le FCE. Seule Sheen (2011 : 91-111) a testé le type 6, dans sa version locale (ciblant les déterminants indéfinis), et elle a montré que ce dernier était plus efficace que la correction directe (type 5). Sur la base d'une seule recherche récente, il est cependant difficile de se prononcer sur l'efficacité des FCE explicites.

Il semble donc que la correction directe soit la plus efficace des FCE implicites. Une hypothèse vraisemblable est qu'exposer l'élève immédiatement à la forme correcte est plus productif que de la lui faire chercher (Bitchener, 2012 ; Chandler, 2003). En outre, la correction directe a l'avantage de fournir à l'élève un énoncé correct (positive evidence) : même s'il ne comprend pas son erreur, il saura qu'il s'agit d'une erreur et il aura été exposé à sa forme correcte.

\section{Les facteurs externes susceptibles de modérer l'efficacité du FCE}

Comme nous le soulignons dans l'introduction, le contexte dans lequel s'opère la régulation a également un impact sur l'efficacité de cette dernière :

D'une part, il est probable que l'effet des feedback adressés à un individu en situation d'apprentissage ou de réalisation d'une tâche dépend de paramètres de contexte (la nature de la tâche, notamment) et de caractéristiques de personnalité. D'autre part, il est probable que l'effet des feedback ou réactions des enseignants fluctue en fonction d'autres conduites d'enseignement. (Crahay, $2007: 59)$

De nombreuses variables extrinsèques au FCE ont un impact sur l'efficacité de celui-ci. On peut mentionner, entre autres, les connaissances (méta)linguistiques de l'élève, sa motivation, son niveau d'interlangue, la tâche relative à la réception des FCE, la présence de syntagmes qui ont fait l'objet d'une correction dans l'input suivant l'exposition aux FCE, le type de structures lexicales ou grammaticales corrigées, la stratégie de correction (globale ou locale). Une revue détaillée de ces variables est disponible dans Hyland \& Hyland (2006) et Ferris (2011).

Deux variables ont une influence importante sur la recherche que nous présentons dans la troisième partie de cet article et dans les pistes de solution que nous proposons. D'une part, les FCE semblent être plus performants lorsqu'ils sont fournis à la suite d'une production rédigée à des fins communicatives (dans le but de produire du sens) plutôt qu'à des fins d'évaluation et d'amélioration exclusivement formelles. Fazio (2001) a en effet montré que si les élèves perçoivent que le message importe peu, les FCE sont moins efficaces, car c'est le retour sur le sens que l'élève veut communiquer qui permet de restructurer l'interlangue et d'y intégrer les nouveaux éléments lexicaux et grammaticaux de façon plus efficace. D'autre part, les FCE apparaissent être plus performants s'ils sont délivrés dans le cadre d'une évaluation formative (Ferris, 2011 : 93) 
. En effet, si l'enseignant recourt aux FCE dans le cadre d'une évaluation certificative, ils risquent d'être perçus comme une (simple) justification de la note finale, alors que les élèves les verront plus comme une aide pédagogique dans le cadre d'une évaluation formative, surtout si cette dernière est suivie d'une évaluation certificative qui lui est similaire (voir 3.3.).

\section{Éléments de contextualisation} pour la provision de FCE dans le cadre d'évaluations formatives. Or, il nous semble que le recours à l'évaluation formative est une pratique minoritaire en $\mathrm{FWB}^{4}$. Cela peut s'expliquer en partie par le fait que les professeurs de langues doivent fréquemment certifier leurs élèves. En effet, comme le montre le tableau 2, le nombre d'évaluations certificatives minimum à administrer aux élèves est considérable, d'autant qu'il faut y ajouter l'évaluation des savoirs grammaticaux, lexicaux et des fonctions langagières.

Tableau 2 - Nombre d'évaluations certificatives minimum requises par année scolaire, examens compris, dans les deux réseaux scolaires principaux de la FWB (Noiroux \& Simons, $2015: 111$ )

\begin{tabular}{|l|l|l|}
\hline & $\begin{array}{l}\text { Communauté française de } \\
\text { Belgique (2002:1-2) }\end{array}$ & $\begin{array}{l}\text { Fédération de l'Enseignement } \\
\text { Secondaire Catholique (2010:9) }\end{array}$ \\
\hline $\begin{array}{l}\text { compréhension à } \\
\text { l'audition }\end{array}$ & 4 & 3 \\
\hline $\begin{array}{l}\text { compréhension à la } \\
\text { lecture }\end{array}$ & 4 & 3 \\
\hline expression écrite & 4 & 3 \\
\hline $\begin{array}{l}\text { expression/ } \\
\text { interaction orale }\end{array}$ & 3 & 3 \\
\hline
\end{tabular}

27 Ces résultats nous font penser qu'il est peu probable que les enseignants prennent le temps de recourir à l'évaluation formative tant ils doivent évaluer certificativement.

Outre la fréquence des évaluations certificatives dans les différentes compétences, il faut relever la nature particulièrement chronophage de l'acte d'évaluation de la compétence écrite. En effet, en FWB, il n'est pas rare que le professeur d'anglais ait plus de cent élèves quand il exerce à temps plein. Par ailleurs, l'administration d'une épreuve certificative portant sur l'expression écrite consomme au moins une période de cours $\left(50^{\prime}\right)$ et la correction de chaque copie d'élève prend un temps considérable.

Enfin, rappelons que l'intérêt de l'évaluation formative réside dans l'exploitation des erreurs de l'apprenant un peu avant l'évaluation certificative, entre le moment où tous les savoirs ont été enseignés et entrainés, et le moment où ils sont évalués 
certificativement. Cela implique donc que l'enseignant doit procéder à la correction de l'intégralité des copies de la classe en peu de temps, difficulté qui complique l'utilisation systématique de l'évaluation formative.

Ces éléments nous conduisent à penser que les professeurs recourent au FCE majoritairement dans le cadre d'expressions écrites certificatives.

31 Puisqu'ils ne peuvent plus améliorer leur note, il est peu probable que tous les élèves - et principalement les élèves faibles - prêtent à leurs erreurs l'attention qu'elles méritent, $\grave{a}$ fortiori quand le dispositif pédagogique mis en place se limite à une brève séance de questions-réponses. Il semble donc nécessaire d'implémenter d'autres dispositifs pour les y inciter (voir point 3.3.).

\section{Recherche exploratoire}

Comme nous l'avons montré dans le premier volet de cet article, les études réalisées sur les différents types de FCE semblent arriver à la conclusion que la correction directe est la plus efficace. Cependant, aucune de ces recherches ne propose des résultats plus fins sur l'efficacité de cette dernière. Autrement dit, rien ne garantit que la correction directe offre un taux de compréhension acceptable. Dans Ferris (1997), la chercheuse conclut que les étudiants sont capables de réviser $73 \%$ des passages soumis à des commentaires portant sur la grammaire et rédigés par l'enseignant. Dans Ferris \& Roberts (2001), les auteurs remarquent que les étudiants dont les erreurs grammaticales sont soulignées arrivent à en réviser $60 \% ; 64 \%$ si elles sont soulignées et codées. Aucune recherche similaire n'a été réalisée pour la correction directe.

\section{Méthodologie}

Nous avons sélectionné une classe d'anglais - première langue étrangère - de $5^{\mathrm{e}}$ année du secondaire général (à savoir l'avant-dernière année de la filière qui mène à l'enseignement supérieur et universitaire), composée d'élèves ( $\mathrm{n}=19)$ âgés de 16 à 18 ans, située dans une école du centre de Liège. Le professeur, en fin de carrière, suit l'approche communicative.

Les élèves ont rédigé une expression écrite certificative corrigée par le professeur titulaire. La tâche consistait en la rédaction d'une critique de film. Ils ont disposé de deux périodes de cours ( 2 fois $50^{\prime}$ ) pour la rédiger et d'un modèle à imiter. Ensuite, le professeur a récolté les copies, les a corrigées (sans avoir été informé de l'objet de la recherche) et nous les a transmises 48 heures plus tard.

Dans un premier temps, nous avons encodé chaque FCE du professeur portant sur une erreur formelle, l'erreur sur laquelle le FCE portait, le type de FCE utilisé, et, le cas échéant, les informations afférentes au FCE (la correction, le code erreur, l'explication). Dans un deuxième temps, à savoir 48 heures après la remise des copies corrigées, nous avons procédé à la récolte des données des élèves. Pour ce faire, nous avons généré, sur la base des erreurs encodées, un questionnaire qui comportait toutes les erreurs que l'enseignant avait corrigées sur la copie de l'élève. Nous l'avons administré à chaque élève de la classe, et ce, pendant une période de cours. Pour chacune des erreurs, nous avons demandé aux élèves s'ils comprenaient l'erreur (cf. tableau 3, "compréhension déclarée"), et, le cas échéant, de fournir la réponse correcte ou, si elle était déjà donnée, d'expliquer 
l'erreur (cf. tableau 3, "compréhension effective"). Les élèves ont rempli le questionnaire sans aide externe ; le seul document qu'ils pouvaient consulter était leur texte corrigé. Une fois le questionnaire complété et rendu, ils ont pu poser toutes les questions qu'ils désiraient à leur professeur et aux chercheurs.

En ce qui concerne le traitement des données, nous avons encodé toutes les réponses des élèves. Ensuite, nous avons analysé toutes les erreurs corrigées par correction directe. Nous les avons classées dans les catégories suivantes:

1. L'élève déclare ne pas comprendre son erreur.

2. L'élève déclare qu'il comprend son erreur. Pour pouvoir l'attester, nous lui avons demandé de l'expliquer. Trois cas de figure se sont produits :

a. L'explication est correcte et prouve sans ambiguïté que l'élève comprend son erreur. Par exemple, dans l'énoncé "He *go [goes] to the USA", nous avons accepté l'explication "+s à la $3^{\mathrm{e}}$ personne du singulier", même si la règle n'est pas complète (elle ne tient pas compte des modifications orthographiques et des verbes être et avoir), car elle confirme que l'élève a compris sur quoi portait l'erreur.

b. L'explication n'est pas correcte. Dans l'énoncé "I['ve known] her *since [for] years", l'élève a expliqué qu'on utilise "for" "car verbe après". Or, on traduit "depuis" par "for" quand il est suivi d'un complément de temps qui rend compte d'une durée, "since" quand le complément de temps fait référence à un moment précis dans le passé.

c. L'explication ne permet pas de déterminer si l'élève a compris son erreur. Dans l'énoncé "The story is about two brothers who *they [/] don't like each other", nous n'avons pas accepté l'explication "grammaire", car elle est bien évidemment trop laconique.

\section{Résultats}

Le tableau suivant synthétise les résultats de la recherche.

Tableau 3 - Compréhension des corrections directes d'une classe de $5^{e}$ secondaire $(n=19)$

\begin{tabular}{|l|l|l|l|l|l|l|}
\hline & & Somme & $\%$ & Moyenne & Médiane & $\begin{array}{l}\text { Écart } \\
\text { type }\end{array}$ \\
\hline & $\begin{array}{l}\text { Nombre de } \\
\text { corrections directes }\end{array}$ & 423 & 100 & 22 & 23 & 6 \\
\cline { 2 - 7 } compréhension déclarée & $\begin{array}{l}\text { L'élève n'a rien déclaré } \\
\text { à propos de son erreur. }\end{array}$ & 34 & 8 & 2 & 1 & 2 \\
\cline { 2 - 7 } & $\begin{array}{l}\text { L'élève a déclaré qu'il } \\
\text { ne comprend pas son } \\
\text { erreur. }\end{array}$ & 135 & 32 & 7 & 7 & 4 \\
\cline { 2 - 7 } & $\begin{array}{l}\text { L'élève a déclaré qu'il } \\
\text { comprend son erreur. }\end{array}$ & 254 & 60 & 13 & 14 & 6 \\
\hline compréhension effective & $\begin{array}{l}\text { L'explication fournie } \\
\text { par l'élève est correcte } \\
\text { et prouve qu'il } \\
\text { comprend son erreur. }\end{array}$ & $(141)$ & $(33)$ & 7 & 7 & 5 \\
\hline
\end{tabular}




\begin{tabular}{|c|c|c|c|c|c|}
\hline $\begin{array}{l}\text { L'explication fournie } \\
\text { par l'élève prouve qu'il } \\
\text { ne comprend pas son } \\
\text { erreur (p. ex. } \\
\text { explication incorrecte). }\end{array}$ & (43) & (10) & 2 & 2 & 2 \\
\hline $\begin{array}{l}\text { L'explication fournie } \\
\text { par l'élève ne permet } \\
\text { pas de prouver qu'il } \\
\text { comprend son erreur } \\
\text { (p. ex. explication } \\
\text { correcte mais trop } \\
\text { vague). }\end{array}$ & $(70)$ & (17) & 4 & 3 & 4 \\
\hline
\end{tabular}

Force est de constater que nous sommes loin d'une compréhension totale des erreurs corrigées par FCE directs puisque seuls $60 \%$ de celles-ci sont déclarées être comprises, 10 $\%$ desquelles s'avèrent être mal comprises (cf. "compréhension effective"). Bien que ces résultats ne soient pas généralisables, corriger au moyen de FCE directs n'est donc pas un gage de compréhension systématique puisqu'on ne peut attester la compréhension de plus de la moitié des erreurs. On note cependant une forte variance entre les élèves (cf. écart type), ce qui suppose d'importantes différences individuelles relatives à la capacité à comprendre (et peut-être à expliquer) leurs erreurs. Le FCE est donc potentiellement inéquitable puisqu'il profite plus à certains élèves qu'à d'autres.

\section{Feedbacks correctifs, dispositif pédagogique et risques d'inégalité}

Certes, toutes les erreurs n'ont pas été comprises, mais notre test se limitait à mesurer la quantité d'erreurs que l'élève pouvait comprendre seul. Il faut cependant être attentif à l'utilisation que font les élèves et le professeur du texte corrigé (figure 1, étape 3) pour identifier si les pratiques de classe réduisent le caractère potentiellement inéquitable du FCE.

Nous n'avons malheureusement pas pu assister à toutes les leçons qui ont précédé et suivi la rédaction de l'expression écrite. Cependant, nous avons procédé à un entretien semistructuré avec l'enseignant pour obtenir les informations qui nous manquaient. De ces données ressort une pratique en partie équitable, et en partie inéquitable.

Les élèves ont rédigé une expression écrite dans un cadre communicatif (voir 2.). En effet, après avoir suivi plusieurs leçons sur la critique de film, ils ont dû en rédiger une sur un film ou un dessin animé de leur choix. Il ne s'agissait donc pas d'un exercice de grammaire déguisé, mais bien d'une production personnelle, porteuse de sens.

En revanche, les FCE ont été délivrés exclusivement dans le cadre d'une expression écrite certificative. Or, comme nous l'avons indiqué précédemment (voir 2.), quand les FCE sont délivrés dans le cadre d'une évaluation certificative, ils ont tendance à être davantage perçus comme une justification de la note (retraits de points pour telle ou telle erreur) que comme un outil d'apprentissage.

Dans le cas de notre recherche exploratoire, les élèves ont eu la possibilité de poser des questions au professeur après avoir reçu leur copie corrigée. Cette séance de questions- 
réponses a duré quinze minutes et s'est arrêtée lorsque les élèves ont cessé de poser des questions. Ce dispositif appelle néanmoins un commentaire : si certains élèves ont pu tirer profit de la brève séance de questions-réponses, nous doutons que celle-ci ait permis à tous les élèves de comprendre leurs corrections. En effet, un élève faible ayant reçu une note basse et une feuille contenant un (très) grand nombre de corrections peut-il comprendre ses nombreuses erreurs en posant seulement quelques questions?

\section{Synthèse et pistes de solution}

Le FCE est un outil indispensable dans l'apprentissage d'une langue étrangère en contexte scolaire. Ce FCE peut prendre différentes formes dont le choix n'est pas sans conséquence pour l'élève. En effet, notre recherche exploratoire a montré que, sans aide externe, les élèves de la classe ciblée n'ont compris que la moitié de leurs erreurs malgré la présence de la forme correcte fournie par l'enseignant. Le travail de ce dernier ne s'arrête donc pas après avoir corrigé les erreurs de l'élève. Ajouter à la correction de l'erreur son explication (cf. feedback explicite) semble être une solution évidente à ce problème. Cependant, nous pressentons que ce mode de correction, extrêmement lourd pour l'enseignant, n'est pas adapté au contexte actuel de la FWB.

Pour résoudre cet épineux problème, différentes solutions sont envisageables.

La première piste de solution, qui nous est inspirée par certaines pratiques d'enseignants, tient compte de la double réalité actuelle en FWB : a) le manque de temps disponible pour l'évaluation formative et $b$ ) le fait que les élèves se focalisent beaucoup plus sur les notes que sur les feedbacks qui leur sont donnés. Ce dispositif consiste à rendre obligatoire l'amélioration de l'expression écrite certificative en instaurant un système de "bonus/ malus" (par exemple de -2 à +2) en fonction de la qualité du travail de correction fourni par l'élève, ceci afin que ce dernier soit "incité" à utiliser et, partant, à comprendre ses FCE. Certes, on peut reprocher à ce système de renforcer une tendance déjà bien/trop marquée chez les élèves qui consiste à "ne travailler que pour des points", mais on peut espérer que, grâce à celui-ci, les élèves s'investiront davantage dans une réflexion sur leurs erreurs et que cette béquille pourra, à terme, être retirée.

La deuxième piste de solution consiste à délivrer aussi les FCE dans le cadre d'expressions écrites formatives, précédant chaque évaluation certificative. De prime abord, cette solution semble relever du bon sens, mais, comme nous l'avons indiqué (voir partie 2), les prescrits légaux actuels en FWB et la nature chronophage de la correction de l'expression écrite ne favorisent guère cette démarche formative et formatrice. Pour que ce dispositif soit effectivement mis en place, il conviendrait de modifier les textes officiels, par exemple en diminuant le nombre d'évaluations certificatives minimum que le professeur doit administrer aux élèves, et/ou en ciblant moins de compétences par année scolaire (Noiroux \& Simons, 2015 : 115). Le pari sous-jacent à cette proposition est qu'en libérant quelque peu les enseignants de la pression de l'évaluation certificative, ils pourront consacrer davantage de temps à l'enseignement/apprentissage et à l'évaluation formative, en ce compris à l'explication des erreurs et à la mise en place d'activités de remédiation adaptées aux types d'erreurs et aux profils des apprenants.

48 Enfin, la troisième piste de solution consiste à orienter davantage les tâches d'écriture vers une finalité moins scolaire que son évaluation telle que la publication d'écrits informatifs, narratifs, incitatifs, argumentatifs... sur le site/dans le journal de l'école, sur 
un blog ou sur une page Facebook. Cette démarche fournirait sans doute davantage de sens à l'acte d'écriture qu'une tâche de simulation, typique de l'approche communicative (Puren, 2006, 2014), et rendrait peut-être les feedbacks correctifs plus significatifs (voir 1.4.). Au sujet de ces derniers, on pourrait faire procéder à des révisions progressives des textes par des pairs plus avancés de la classe (ou d'une autre classe), voire par des locuteurs natifs du même âge dans le cadre d'un apprentissage "en tandem" (Simons, Dahmen, Halink \& Van Hoof, 2006) en leur faisant utiliser, par exemple, la fonction "suivi des modifications". Dans ce cas de figure, la tâche du professeur consisterait à (in)valider les corrections ou suggestions de modifications faites par les pairs plus avancés, et, éventuellement, à expliciter les corrections directes implicites. Relevons que cette explicitation pourrait être non seulement bénéfique pour l'élève corrigé, mais peut-être aussi pour l'élève correcteur car ce dernier peut avoir une perception intuitive de la forme erronée, sans pour autant être capable de l'expliquer. Ce troisième type de solution, qui n'est pas incompatible avec les deux premiers, inscrirait davantage le cours de langue dans l'approche actionnelle où l'élève apprend progressivement à agir, à travers la langue étrangère, comme un agent social, ce qui pourrait lui être demandé au terme de ses études.

\section{BIBLIOGRAPHIE}

Allal, L. (2007). "Régulation des apprentissages : orientations conceptuelles pour la recherche et la pratique en éducation". In Allal, L. \& Mottier Lopez, L. (dir.), Régulation des apprentissages en situation scolaire et en formation. Bruxelles : De Boeck Supérieur.

Bitchener, J. (2008). "Evidence in support of written corrective feedback". Journal of Second Language Writing, vol. 17, $\mathrm{n}^{\circ}$ 2, pp. 102-118.

Bitchener, J. (2012). "A reflection on 'the language learning potential' of written CF". Journal of Second Language Writing, vol. 21, $\mathrm{n}^{\circ} 4$, pp. 348-363.

Bitchener, J., Young, S. \& Cameron, D. (2005). "The effect of different types of corrective feedback on ESL student writing". Journal of Second Language Writing, vol. 14, n 3, pp. 191-205.

Chandler, J. (2003). "The efficacy of various kinds of error feedback for improvement in the accuracy and fluency of L2 student writing". Journal of Second Language Writing, vol. 12, n 3, pp. 267-296.

Communauté française de Belgique. (2002). Circulaire sur l'évaluation. Enseignement des langues germaniques à la Communauté française. Bruxelles: Communauté française de Belgique.

Crahay, M. (2007). "Feedback de l'enseignant et apprentissage des élèves : revue critique de la littérature de recherche". In Allal, L. \& Mottier Lopez, L. (dir.), Régulation des apprentissages en situation scolaire et en formation. Bruxelles : De Boeck Supérieur.

Ellis, R., Loewen, S. \& Erlam, R. (2006). "Implicit and explicit corrective feedback and the acquisition of L2 grammar". Studies in Second Language Acquisition, vol. 28, n², pp. 339-339. 
Fazio, L. L. (2001). "The effect of corrections and commentaries on the journal writing accuracy of minority-and majority-language students". Journal of Second Language Writing, vol. 10, $\mathrm{n}^{\circ}$ 4, pp. 235-249.

Fédération de l'Enseignement Secondaire Catholique. (2010). Note sur l'évaluation - Humanités générales et technologiques. Bruxelles: Fédération de l'Enseignement Secondaire Catholique.

Ferris, D. R. (1997). "The influence of teacher commentary on student revision". TESOL Quartely, vol. 29, pp. 33-53.

Ferris, D. R. (1999). "The case for grammar correction in L2 writing classes: A response to Truscott (1996)". Journal of Second Language Writing, vol. 8, n 1, pp. 1-11.

Ferris, D. R. (2004). "The "Grammar Correction" Debate in L2 Writing: Where are we, and where do we go from here? (and what do we do in the meantime...?)". Journal of Second Language Writing, vol. $13, n^{\circ} 1$, pp. 49-62.

Ferris, D. R. (2006). "Does error feedback help student writers? New evidence on short- and longterm effects of written error corrections.". In Hyland, K. \& Hyland, F. (dir.), Feedback in Second Language Writing: Contexts and Issues. Cambridge : Cambridge University Press.

Ferris, D. R. (2011). Treatment of Error in Second Language Student Writing (Second ed.). Ann Arbor: University of Michigan Press.

Ferris, D. R. \& Roberts, B. (2001). "Error feedback in L2 writing classes: How explicit does it need to be?". Journal of Second Language Writing, vol. 10, $n^{\circ} 3$, pp. 161-184.

Gass, S. M. (1988). "Integrating Research Areas: A Framework for Second Language Studies". Applied linguistics, vol. 9, $\mathrm{n}^{\circ}$ 2, pp. 198-217.

Hyland, K. \& Hyland, F. (dir.). (2006). Feedback in Second Language Writing: Contexts and Issues. Cambridge: Cambridge University Press.

Noiroux, K. \& Simons, G. (2015). "La copie d'expression écrite certificative : un support équitable ?". Spirale, vol. 55, pp. 105-117.

Puren, C. (2006). L'évaluation a-t-elle encore un sens ? http://www.aplv-languesmodernes.org// spip.php?article36

Puren, C. (2014). Entre approche communicative et perspective actionnelle: prolongement, rupture, complémentarité? Université de Liège. Liège. https://youtu.be/3mBPJGN9P_I

Russell, J. \& Spada, N. (2006). "The effectiveness of corrective feedback for the acquisition of L2 grammar". In Norris, J. (dir.), Synthesizing Research on Language Learning and Teaching. Philadelphia : John Benjamins.

Schmidt, R. (1990). "The Role of Consciousness in Second Language Learning". Applied linguistics, vol. $11, \mathrm{n}^{\circ} 2$, pp. 129-158.

Schmidt, R. (dir.). (1995). Attention and awareness in foreign language learning. Honolulu, $\mathrm{Hl}$ : University of Hawaii Press.

Schmidt, R. (2001). "Attention". In Robinson, P. (dir.), Cognition and Second Language Instruction. Cambridge : Cambridge University Press.

Sheen, Y. (2010). "Differential effects of oral and written corrective feedback in the ESL classroom". Studies in Second Language Acquisition, vol. 32, n², pp. 201-234.

Sheen, Y. (2011). Corrective Feedback, Individual Differences and Second Language Learning. New York: Springer. 
Sheen, Y. \& Ellis, R. (2011). "Corrective Feedback in Language Teaching". In Hinkel, E. (dir.), Handbook of Research in Second Language Teaching and Learning (Vol. II) : Routledge.

Truscott, J. (1996). "The Case Against Grammar Correction in L2 Writing Classes". Language Learning, vol. 46, $\mathrm{n}^{\circ}$ 2, pp. 327-369.

Van Beuningen, C. G., De Jong, N. H. \& Kuiken, F. (2008). "The Effect of Direct and Indirect Corrective Feedback on L2 Learners' Written Accuracy". ITL-Review of Applied Linguistics, vol. 156, pp. 279-296.

Van Beuningen, C. G., De Jong, N. H. \& Kuiken, F. (2012). "Evidence on the Effectiveness of Comprehensive Error Correction in Second Language Writing". Language Learning, vol. 62, $\mathrm{n}^{\circ} 1$, pp. 1-41.

White, L. (1991). "Adverb placement in second language acquisition: Some effects of positive and negative evidence in the classroom". Second Language Research, vol. 7, n², pp. 133-161.

Zhao, Y., Song, L. \& Cherrington, R. (2013). "Interlangue". In Byram, M. \& Hu, A. (dir.), Routledge Encyclopedia of Language Teaching and Learning (2nd ed.). London : Routledge.

\section{CD-ROM}

Simons, G., Dahmen, M., Halink, R. \& Van Hoof, F. (2006). L'apprentissage en tandem. Apprendre une langue étrangère de et avec l'autre. Bruxelles: Administration générale de l'Enseignement et de la Recherche scientifique. Service général des Affaires pédagogiques et du Pilotage du réseau d'enseignement organisé par la Communauté française, Talenacademie Nederland.

\section{NOTES}

1. "[The interlanguage] refers to both the internal system that a learner has constructed at a single point in time ('an interlanguage') and to the series of interconnected systems that characterise the learner's progress over time ('interlanguage' or 'the interlanguage continuum') [...]" (Zhao, Song \& Cherrington, $2013: 356$ ).

2. Cela peut aussi être " ortho" pour orthographe, " ponct" pour ponctuation, " synt" pour syntaxe, etc.

3. Nous écartons bien sûr le cas de la surcharge de travail ou encore celui d'un investissement minimaliste de l'enseignant par rapport à l'acte de régulation de l'apprentissage.

4. Nous faisons également référence à notre expérience professionnelle en tant qu'enseignant et en tant que formateur d'enseignants (voir la notice sur les auteurs) qui nous permet d'entretenir des contacts soutenus avec de très nombreux enseignants de langues en FWB (près de 200).

\section{RÉSUMÉS}

L'enseignant de langues étrangères dispose de nombreux supports pour soutenir l'apprentissage de ses élèves. Cette contribution se centre sur un support particulier : le texte rédigé par l'élève et corrigé par l'enseignant. Les corrections ont entre autres pour but d'améliorer la maitrise 
linguistique de la langue étrangère de l'élève. Mais l'enseignant atteint-il toujours ce but ? Les résultats de la recherche exploratoire que nous avons menée dans une classe d'anglais langue étrangère de Belgique francophone montrent que tous les élèves de la classe ciblée ne sont pas à même de comprendre la majorité de leurs erreurs, et que le dispositif didactique mis en place par l'enseignant ne permet pas nécessairement de pallier le caractère potientiellement inéquitable de la correction des erreurs.

The foreign language teacher has a lot of teaching aids at his disposal to support his students' learning. This paper focuses on one particular aid: the text written by the student and corrected by the teacher. The corrections aim, among other things, at improving the student's linguistic command of the foreign language. But does the teacher always reach this goal? The results of the exploratory study that we carried out in a foreign language class in Belgium's French-speaking community show that not all the students in the targeted class manage to understand their mistakes, and the didactic device that the teacher implemented does not necessarily allow overcoming the inequitable nature of the error correction process.

\section{INDEX}

Keywords : writing, corrective feedback, English as a second language, Federation WalloniaBrussels, summative assessment, formative assessment, equality, tentative solutions

Mots-clés : feedback correctif, expression écrite, anglais/langue étrangère, Fédération Wallonie-Bruxelles, évaluation certificative, évaluation formative, équité, pistes de solution

\section{AUTEURS}

\section{KEVIN NOIROUX}

Université de Liège, Belgique

Kevin Noiroux est boursier de doctorat NON FRIA, attaché au Service de didactique des langues modernes de l'Université de Liège. Sa thèse porte sur la correction des erreurs en anglais langue étrangère dans le contexte de l'enseignement secondaire supérieur. Il enseigne actuellement l'anglais et le néerlandais dans l'enseignement secondaire, supérieur de type court, et universitaire.

Courriel : k.noiroux[at]ulg.ac.be.

Adresse : Université de Liège, Service de didactique des langues modernes (A2), Place Cockerill 3-5 (Bât. A2), 4000 Liège.

\section{GERMAIN SIMONS}

Université de Liège, Belgique

Germain Simons est Professeur de didactique des langues modernes à la Faculté de Philosophie et Lettres de l'Université de Liège (ULg). Il est titulaire du cours de didactique des langues modernes destiné aux étudiants de l'Agrégation de l'Enseignement Secondaire Supérieur et du Master à finalité didactique, et est responsable du cours de didactique professionnelle des langues modernes organisé dans le cadre du Certificat d'Aptitude Pédagogique Approprié à l'Enseignement Supérieur. Depuis 2013, il est Président du Cifen (Centre interfacultaire de formation des enseignants) de l'ULg. Courriel : g.simons[at]ulg.ac.be. Adresse : Université de Liège, Service de didactique des langues modernes, Place Cockerill 3-5 (Bât. A2), 4000 Liège. 